\title{
One-dimensional ultrasound receive array using spectrally encoded optical detection
}

\author{
T. Buma ${ }^{a)}$ \\ Center for Ultrafast Optical Science, University of Michigan, Ann Arbor, Michigan 48109-2099 \\ M. O’Donnell \\ Department of Biomedical Engineering, University of Michigan, Ann Arbor, Michigan 48109-2099
}

(Received 7 September 2004; accepted 25 October 2004)

\begin{abstract}
An ultrafast Ti:Sapphire laser and a Sagnac interferometer are combined for optical detection of ultrasound. Distinct spatial positions are probed simultaneously by different wavelengths within the broadband laser. Ultrasonic signals from each probe position are derived from the spectrum of the reflected light. The same single-mode fiber delivers incident and reflected light. A one-dimensional receive array is demonstrated by measuring the acoustic field of a spherically focused piezoelectric transducer. This is a promising form of parallel detection for miniaturized high-frequency ultrasonic arrays. (C) 2004 American Institute of Physics. [DOI: 10.1063/1.1835000]
\end{abstract}

Piezoelectric arrays for high-frequency ultrasound imaging have been difficult to fabricate due to the extremely small element sizes and electrical connections. ${ }^{1}$ Optical detection of ultrasound is a promising alternative to piezoelectricity for high-frequency applications. ${ }^{2,3}$ An array can be synthesized by rapidly scanning a focused laser beam over the region of interest. An alternative to laser scanning is to perform parallel detection, where all receive elements operate simultaneously.

Tearney ${ }^{4}$ has demonstrated spectral encoding of broadband light for parallel detection in a confocal microscopy probe. A single-mode fiber delivers broadband light to a diffraction grating, where the spectrally dispersed light allows different wavelengths to probe different positions. We adapt this technique for optical detection of ultrasound. Most optical methods use an interferometer stabilized for proper operation at a specific laser wavelength. The Sagnac interferometer does not require such stabilization, making it compatible with broadband optical sources. ${ }^{5}$ However, proper operation requires the optical source coherence length to be shorter than the differential path length of the interferometer. $^{6}$ Furthermore, the acoustic detection bandwidth $^{7}$ is inversely proportional to the differential path length. The extremely short coherence length of an ultrafast laser pulse permits a small differential path length suitable for detecting high-frequency ultrasound.

The overall setup is shown in Fig. 1. The optical source is an argon-pumped ultrafast Ti:Sapphire oscillator producing $70 \mathrm{fs}$ pulses at a $76 \mathrm{MHz}$ repetition rate. The laser spectrum is peaked at $795 \mathrm{~nm}$ with a $15 \mathrm{~nm}$ bandwidth. A first pass through the Sagnac interferometer imparts a phase bias to the broadband light. The laser is spectrally dispersed by a ruled diffraction grating blazed for $750 \mathrm{~nm}$ with 1200 lines $/ \mathrm{mm}$. An achromatic lens focuses the different wavelengths on an aluminum-coated pellicle, a $5 \mu \mathrm{m}$ thick nitrocellulose membrane. The pellicle, acting as the acoustic sensing surface, is attached to a water tank containing a 15 $\mathrm{MHz}$ piezoelectric transducer. The pellicle is positioned at the focus of the transducer, which has an $f /$ number of 1.5 and a focal length of $18 \mathrm{~mm}$. The pellicle motion phase



modulates the reflected light, which is recombined by the diffraction grating and sent back to the interferometer. The second pass through the Sagnac interferometer demodulates the reflected light, producing the ultrasonic signal.

The Sagnac interferometer, shown in Fig. 2(a), can be understood by tracing the path of a single incident laser pulse. The incident pulse is horizontally polarized and transmitted by the polarizing beam splitter (PBS). The pulse is then split into two pulses by a 50/50 nonpolarizing beam splitter (NPBS). The pulse taking the long arm is delayed by a time $\tau=4 \mathrm{~ns}$ relative to the pulse taking the short arm. A second 50/50 NPBS recombines the two pulses, which then pass through a Faraday rotator (FR). A half-wave plate optimizes the orientation of the polarization of the two pulses before entering a $1 \mathrm{~m}$ long polarization-maintaining singlemode fiber. The fiber outputs horizontally polarized light to the diffraction grating in Fig. 1.

After reflecting off the pellicle, the two laser pulses returning from the diffraction grating travel back through the optical fiber. The FR changes their polarization from horizontal to vertical. The light is then split by the second NPBS, where one set of pulses is delayed with respect to the other by $\tau=4 \mathrm{~ns}$. The two sets of pulses are recombined by the first NPBS, producing three output pulses. The PBS reflects the



FIG. 1. Spectral encoding of the broadband laser. A diffraction grating disperses the laser light, where different wavelengths probe distinct positions on the acoustic sensing surface. 


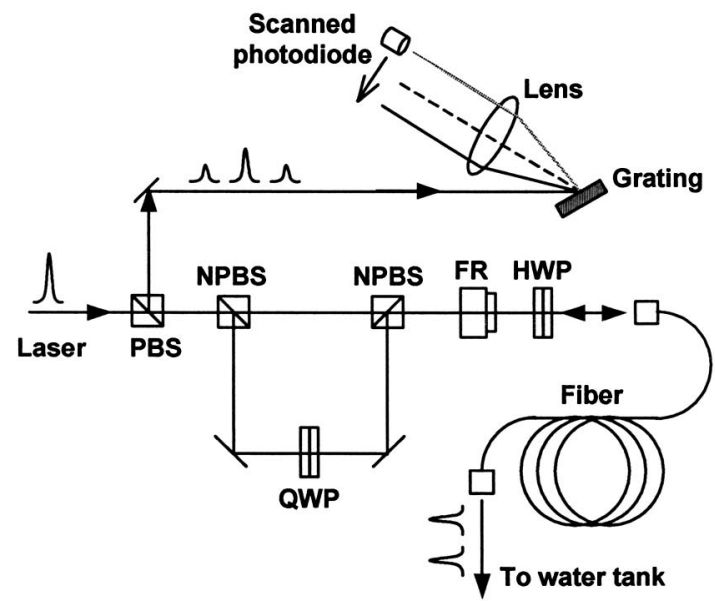

(a)
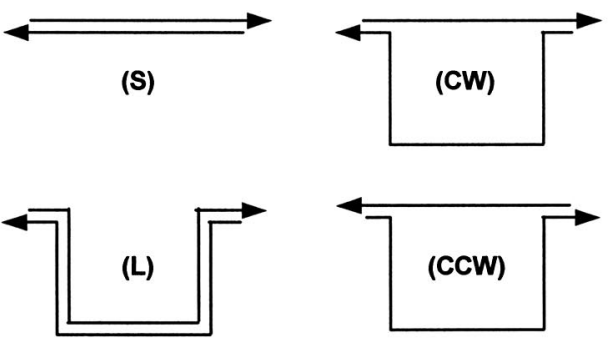

(b)

FIG. 2. Sagnac interferometer. (a) The QWP and FR provide the $\pi / 2$ phase bias required for proper operation. The ultrasonic signals from all the receive elements are carried by the same optical fiber. (b) Possible light paths in the interferometer.

light to a second diffraction grating. This "decoding" grating is followed by an achromatic lens focusing the dispersed wavelengths onto the photodetector plane.

The first and third pulses arriving at the photodetector are due to the laser traversing only the short and long arms, respectively. These paths are labeled in Fig. 2(b) as " $\mathrm{S}$ " and "L". The middle pulse is the sum of two pulses sharing a common path involving both the short and long arms. One pulse travels in the clockwise ("CW") direction while the other pulse takes the counter-clockwise ("CCW") path. Their interference produces the interferometer signal. The long arm contains a quarter-wave plate (QWP) oriented at zero degrees with respect to the horizontal polarization. The QWP therefore does not affect the polarization. The $\mathrm{CW}$ and $\mathrm{CCW}$ paths require the laser to travel along the fast and slow axes of the QWP, respectively. This produces the $\pi / 2$ phase bias between the two paths necessary for proper operation of the Sagnac interferometer. ${ }^{8}$

Ideally, the laser spectrum is measured by a high-speed photodiode array. As a proof of principle, a single-amplified Si photodiode is scanned across the laser spectrum. The photodiode has an active diameter of $50 \mu \mathrm{m}$, a responsivity of $500 \mathrm{~V} / \mathrm{W}$, and an electrical bandwidth of $1.5 \mathrm{GHz}$. The mode-locked laser pulses are effectively a comb function, so the acoustic sampling rate is equal to the laser repetition rate of $76 \mathrm{MHz}$. The ultrasound signal is an extremely small amplitude modulation riding on top of the optical pulse train. The ultrasound signal is obtained by sending the raw photodetector output through a passive $35 \mathrm{MHz}$ low-pass filter followed by a $60 \mathrm{~dB}$ amplifier. The resulting signal is aver-

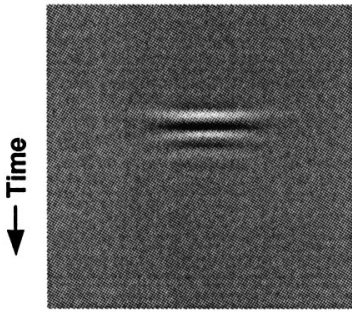

(a)

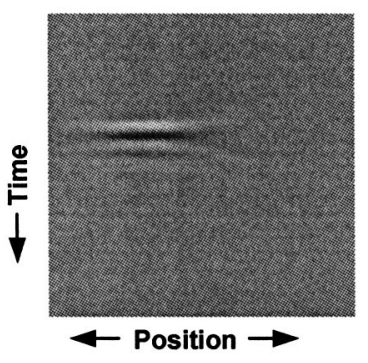

(c)

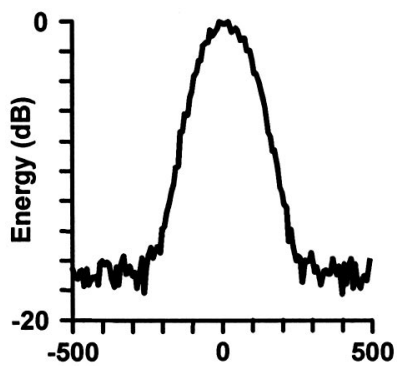

(b)

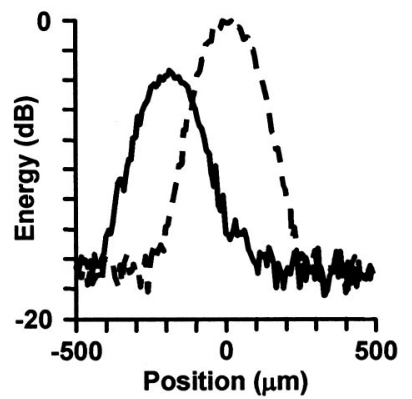

(d)
FIG. 3. Acoustic signals measured with spectral encoding. (a) Wavefield plot and (b) beam plot of the transducer focus at the center of the array. (c) Wavefield plot and (d) beam plot of the transducer focus shifted by $250 \mu \mathrm{m}$. For comparison, the beam plot from (b) is shown by the dashed curve in (d).

aged 16 times before storage by a digital oscilloscope.

The spectral encoding and decoding of the laser are performed with identical gratings. However, the lenses preceding the pellicle and photodetector have focal lengths of 40 $\mathrm{mm}$ and $260 \mathrm{~mm}$, respectively. The spatial extent of the laser spectrum on the photodetector is therefore a factor of 6.5 times larger than on the pellicle. This allows an ultrasonic receive element to be imaged onto a larger photodiode. The photodiode is scanned across a total distance of $6.5 \mathrm{~mm}$ in 50 $\mu \mathrm{m}$ increments. This corresponds to a $1 \mathrm{~mm}$ long ultrasound array with an element pitch of 8 um.

The lateral position of the transducer focus is approximately in the center of the receive array. The average optical power incident on the pellicle is $7.5 \mathrm{~mW}$. The acquired signals are displayed as a wavefield plot in Fig. 3(a). The $x$ axis is element location spanning a $1 \mathrm{~mm}$ distance, while the $y$ axis is time covering a $1 \mu \mathrm{s}$ window. All wavefield plots in this letter are displayed over a linear gray scale, where white and black represent maximum compression and rarefaction, respectively, while midgray represents zero pressure. Each column is the signal measured at a particular location. The transducer focus is clearly visible in the wavefield plot. Figure 3(b) shows a beam plot obtained by computing the energy contained in each column of Fig. 3(a). The $-3 \mathrm{~dB}$ width of the beam plot is $184 \mu \mathrm{m}$.

Shifting the transducer laterally by $250 \mu \mathrm{m}$ produces the wavefield plot shown in Fig. 3(c), shown with the same dynamic range as Fig. 3(a). The reduced strength of the transducer focus is due to the amplitude profile of the laser spectrum, peaked in the center of the array. In other words, the array is apodized by the shape of the laser spectrum. The beam plot in Fig. 3(d) clearly shows the lower signal strength of the shifted transducer focus. This apodization helps explain why the beam plot is peaked less than $250 \mu \mathrm{m}$ away from the array center. 


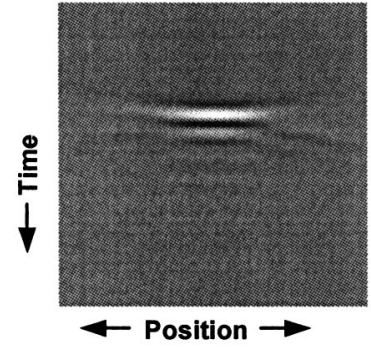

(a)

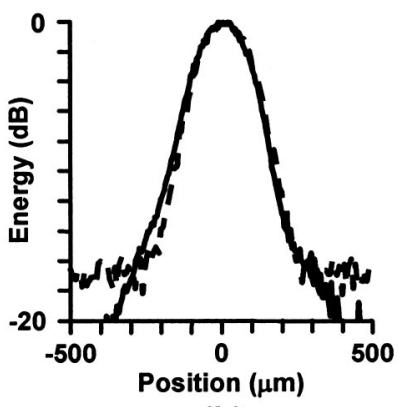

(b)
FIG. 4. Acoustic signals measured without spectral encoding. A single point detector is scanned across the pellicle. (a) Wavefield plot and (b) beam plot of the transducer focus. For comparison, the beam plot from the spectrally encoded data is shown by the dashed curve in (b).

The spectrally encoded array was validated by comparison to a scanned single point detector, where diffraction gratings are not used to disperse the laser. Both the optical fiber and small achromat are mounted on a translation stage to scan the focused light across the pellicle. The focal spot size on the pellicle is estimated to be $11 \mu \mathrm{m}$, much smaller than the peak acoustic wavelength of $100 \mu \mathrm{m}$. The laser was scanned across the pellicle in $5 \mu \mathrm{m}$ increments over a total distance of $1 \mathrm{~mm}$. The transducer focus is positioned approximately in the center of the scanned region. The optical power incident on the pellicle is about $3 \mathrm{~mW}$. The output from the stationary photodiode is sent through a low-pass filter and a $30 \mathrm{~dB}$ amplifier before signal averaging on the digital oscilloscope. The wavefield and beam plots produced by the scanned point detector are shown in Figs. 4(a) and 4(b), respectively. The dashed line in Fig. 4(b) is the beam plot from Fig. 3(b). The close agreement between the two plots suggests that the spectrally encoded array is properly measuring the spatial profile of the acoustic field.

The greater signal-to-noise ratio of the wavefield plot in Fig. 4(a) is largely due to the higher optical power probing a particular point on the pellicle. The effective number of resolvable spectral components in the first-diffraction order is given by: ${ }^{4}$

$$
N=\frac{D \Delta \lambda}{\Lambda \lambda_{0}},
$$

where $D$ is the diameter of the laser beam, $\Lambda$ is the grating line spacing, $\Delta \lambda$ is the optical bandwidth, and $\lambda_{0}$ is the center optical wavelength. The beam diameter is measured to be $3.1 \mathrm{~mm}$, resulting in $N=70$ effective elements in the detection array. This means a receive element is operating with roughly $100 \mu \mathrm{W}$ of light, over an order of magnitude less than the $3 \mathrm{~mW}$ used with the single point detector. Furthermore, the optical power reaching the photodiode was several times lower for the spectral encoding setup. This is probably because a slight misalignment between the grating, lens, and pellicle can significantly degrade the fiber-coupling efficiency of light reflected from the pellicle.

The effective size of an ultrasonic receive element is given by the focal spot of a particular wavelength on the pellicle. Since the lens is working at approximately $f / 13$, the focal spot size on the pellicle is roughly $11 \mu \mathrm{m}$. This is sufficient for a linear array operating up to $130 \mathrm{MHz}$. Figure 4(b) suggests that the spatial sampling of the array is not degraded by an overly large photodiode. The element count decreases for a smaller diameter input beam, which would occur for an in vivo fiber probe. Broader optical bandwidth is then necessary to produce a sufficiently large number of elements.

Future work involves a fiber-optic version of this interferometer in combination with a fiber-based amplified spontaneous emission (ASE) broadband source. An ASE source will also increase the acoustic detection bandwidth for our system, currently limited by the $76 \mathrm{MHz}$ repetition rate of our laser. A high-speed photodiode array will be used to perform true parallel detection. However, a pair of arrays may be required for the balanced detection of the incoherent light. Miniaturizing the diffraction grating and lens is a major issue, although Pitris ${ }^{9}$ has demonstrated a promising technique using a "grism". Combining this technique with resonant optical detection ${ }^{10}$ may provide high sensitivity, high-frequency ultrasound arrays addressed in parallel with a single fiber. The challenge also remains to integrate laser generation and detection systems to form a complete ultrasonic imaging probe. Nevertheless, we believe that these results show the potential of spectrally encoded optical detection in producing arrays for high-frequency ultrasound imaging.

${ }^{1}$ T. A. Ritter, T. R. Shrout, R. Tutwiler, and K. K. Shung, IEEE Trans. Ultrason. Ferroelectr. Freq. Control 49, 217 (2002).

${ }^{2}$ J. D. Hamilton and M. O'Donnell, IEEE Trans. Ultrason. Ferroelectr. Freq. Control 45, 216 (1998).

${ }^{3}$ P. C. Beard and T. N. Mills, Proc. IEEE 88, 1183 (2000).

${ }^{4}$ G. J. Tearney, R. H. Webb, and B. E. Bouma, Opt. Lett. 23, 1152 (1998).

${ }^{5}$ D. H. Hurley, and O. B. Wright, Opt. Lett. 24, 1305 (1999).

${ }^{6}$ J. E. Bowers, Appl. Phys. Lett. 41, 231 (1982).

${ }^{7}$ T. S. Jang, S. S. Lee, I. B. Kwon, W. J. Lee, and J. J. Lee, IEEE Trans. Ultrason. Ferroelectr. Freq. Control 49, 767 (2002).

${ }^{8}$ J. J. Alcoz, C. E. Duffer, and S. Nair, Proc. IEEE 84, 639 (1996).

${ }^{9}$ C. Pitris, B. E. Bouma, M. Shiskov, and G. J. Tearney, Opt. Express 11, 120 (2003).

${ }^{10}$ J. D. Hamilton, T. Buma, M. Spisar, and M. O'Donnell, IEEE Trans. Ultrason. Ferroelectr. Freq. Control 47, 160 (2000). 